\title{
A Case of Delayed Spontaneous Aneurismal Rupture after Ear Surgery Under General Anesthesia
}

\author{
Kyung Seok Lee ${ }^{1}$, Jong Min Lee ${ }^{2}$, Eun Jung Lee ${ }^{1}$ and Yong Joo Yoon ${ }^{1}$ \\ ${ }^{1}$ Department of Otolaryngology-Head and Neck Surgery, Chonbuk National University School of Medicine, Jeonju, \\ ${ }^{2}$ Department of Otolaryngology, Presbyterian Medical Center, Jeonju, Korea
}

$\begin{array}{ll}\text { Received } & \text { December 28, } 2011 \\ \text { Revised } & \text { April 1, 2012 }\end{array}$

Accepted April 21, 2012
Many complications have been reported after ear surgery to treat chronic inflammation. These complications include facial nerve paralysis, perichondritis, injury of the dura or of the sigmoid sinus, cyst formation or mucocele in the healed mastoid cavity, and the recurrence of cholesteatoma, granulation tissue, or otorrhea. It might be believed that there could be no relation between ear surgery and spontaneous aneurysmal rupture, and only one other case of spontaneous aneurysmal rupture after ear surgery under general anesthesia has been previously reported in Korea. However, recently, the authors encountered a case of delayed spontaneous aneurysmal rupture 3 weeks after surgery. No problem was experienced during the operation, and it is suspected that an unidentified pre-existing aneurysm was responsible for the intracerebral hemorrhage.

Korean J Audiol 2012;16:83-86

KEY WORDS: Intracerebral hemorrhage · Otologic surgical procedure · Complications · Aneurysm.

\section{Introduction}

Ear surgery is difficult because the operation field contains many highly complicated, delicate structures, and as a result, many complications can arise. ${ }^{1)}$ However, spontaneous aneurysmal rupture is generally not considered a complication of ear surgery to correct chronic otitis media under general anesthesia. On the other hand, many cases of spontaneous aneurysmal rupture have been reported after surgery under general anesthesia, and the majority of these reports refer to a relation between aneurysmal rupture and hemodynamic changes under general anesthesia.

Here, we describe a case of aneurysmal rupture after ear surgery under general anesthesia. This case emphasizes the need for awareness of severe delayed complication after ear surgery.

\section{Case Report}

A 54-year-old woman visited our department due to bilateral hearing impairment and intermittent otorrhea without dizziness of protracted duration. She did not have tinnitus or otalgia, but complained of recent symptom aggravation. Further- more, she had no known history of medical or surgical problems, except that she was a chronic hepatitis B carrier.

During physical and radiological examinations, a yellowish purulent discharge was noted in the right ear canal and attic destruction was identified. The left ear drum was thinned inferiorly and in a retracted state (Fig. 1), and the Weber test showed left deviation. Pure tone audiometry showed air conduction threshold of $72 \mathrm{~dB} \mathrm{HL}$ and bone conduction threshold of 28 $\mathrm{dB}$ HL for the right ear and air conduction threshold of $50 \mathrm{~dB}$ $\mathrm{HL}$ and bone conduction threshold of $20 \mathrm{~dB}$ HL for the left. Auditory brainstem response testing showed peak waves at $70 \mathrm{dBnHL}$ on the right and at $50 \mathrm{dBnHL}$ on the left. A temporal bone computed tomographic (CT) scan showed sclerosis of mastoid bone and soft tissue densities in the middle ear, attic area, and mastoid cavity on the right side (Fig. 2), but ossicles and other inner ear structures, and the external auditory canal were relatively healthy. Other routine laboratory tests were within normal ranges. However, no evaluation of cerebrovascular structures was performed.

Two days after admission, right side canal wall up mastoidectomy with type I tympanoplasty was performed under general anesthesia. The dura and sigmoid sinus were not exposed 
during surgery. At about 2 hours after surgery, the patient complained of a headache and a rigid sensation in upper and lower extremities. At this time, her blood pressure was elevated at $170 / 100$. Mentally, she was mildly drowsy and oxygen saturation had fallen to $80 \%$. Accordingly, she was placed on mask ventilation, and our department of internal medicine was consulted regarding management. Endo-tracheal intubation was decided upon but not instituted because her condition recovered to near normal within an hour. Department of neurosurgery was then asked to undertake a proper evaluation. However, brain CT returned non-specific findings. Subsequently, the patient was followed a normal recovery course and pattern. Nevertheless, we recommended further evaluation, particularly brain magnetic resonance image, but the patient and her family rejected the idea. Accordingly, regular discharge was ordered 1 week after surgery.

Two weeks after discharge, the patient visited our emergency room with a severe headache and a mental status of stupor. Emergent brain CT and angiography was performed and a subarachnoid hemorrhage (SAH) from a ruptured anterior intercommunicating artery was visualized (Fig. 3). Emergent treph- ination was performed, and subsequently, she was admitted to our neurologic intensive care unit. Nevertheless, intracranial bleeding persisted at follow-up brain CT 2 days later (Fig. 4). The patient expired 1 week after this second admission without recovering consciousness.

\section{Discussion}

A close correlation has been established between the site of

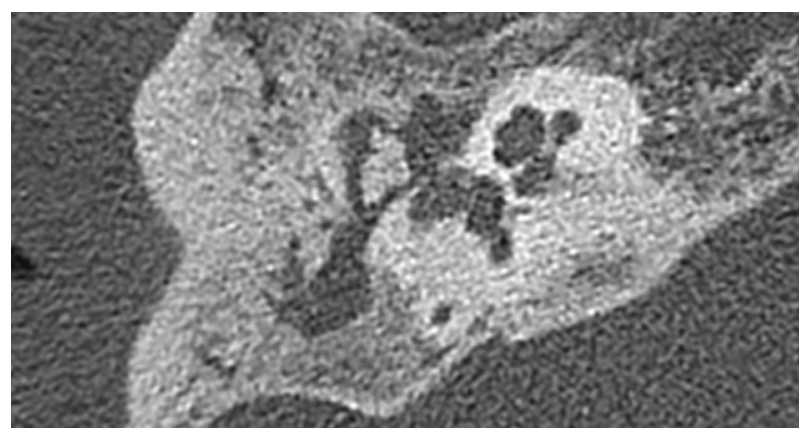

Fig. 2. Temporal bone CT findings. Both images show soft tissue density in the middle ear, attic area, and mastoid cavity.
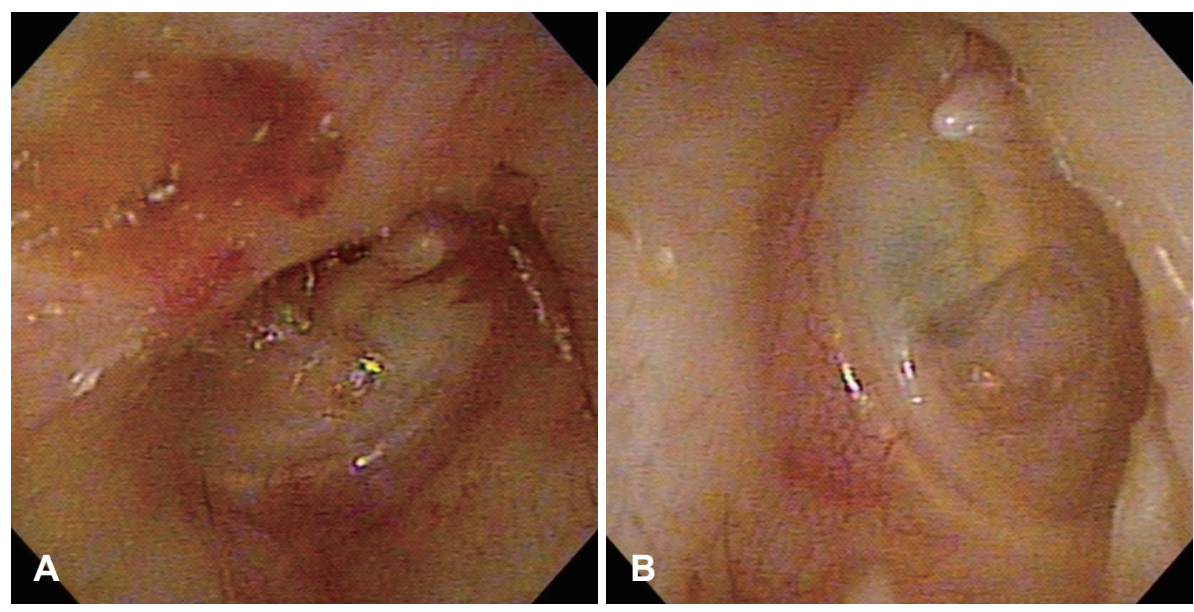

Fig. 1. Otoscopic findings. A: Shows a yellowish mucoid discharge and attic destruction (Rt). B: Shows thinning and retraction of the ear drum (Lt).
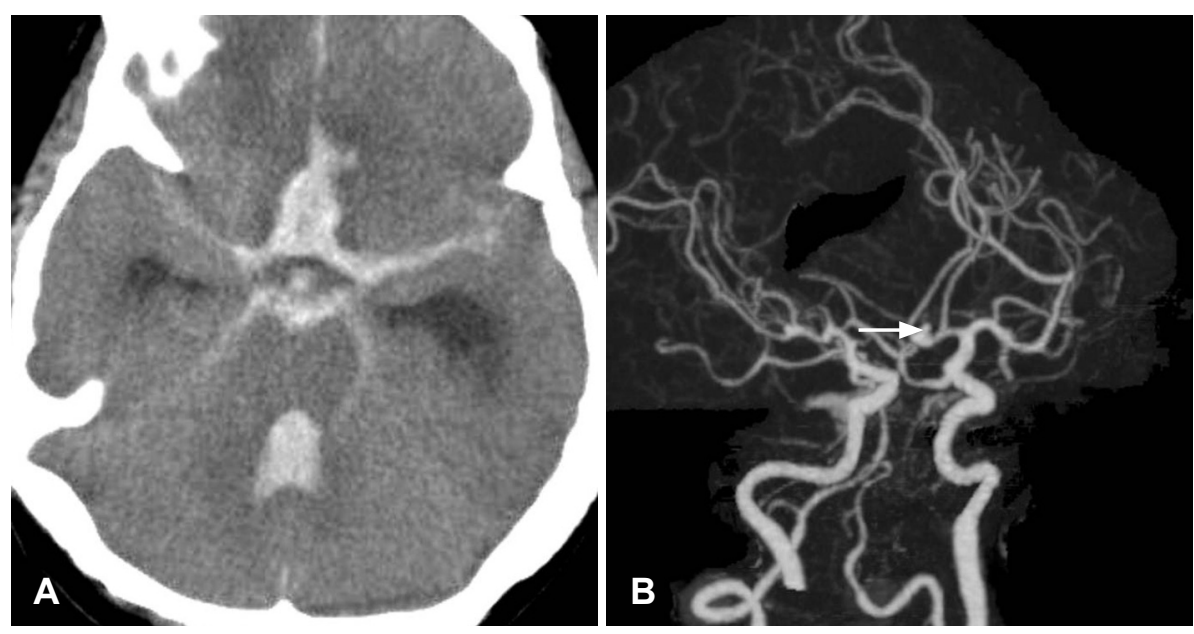

Fig. 3. Brain CT with angiogram findings. A: Shows a hemorrhage in subarachnoid space and intraventricular area. B: Shows a small aneurysm of the anterior communicating artery in Willis circle (white arrow).

84 Korean J Audiol 2012;16:83-86 


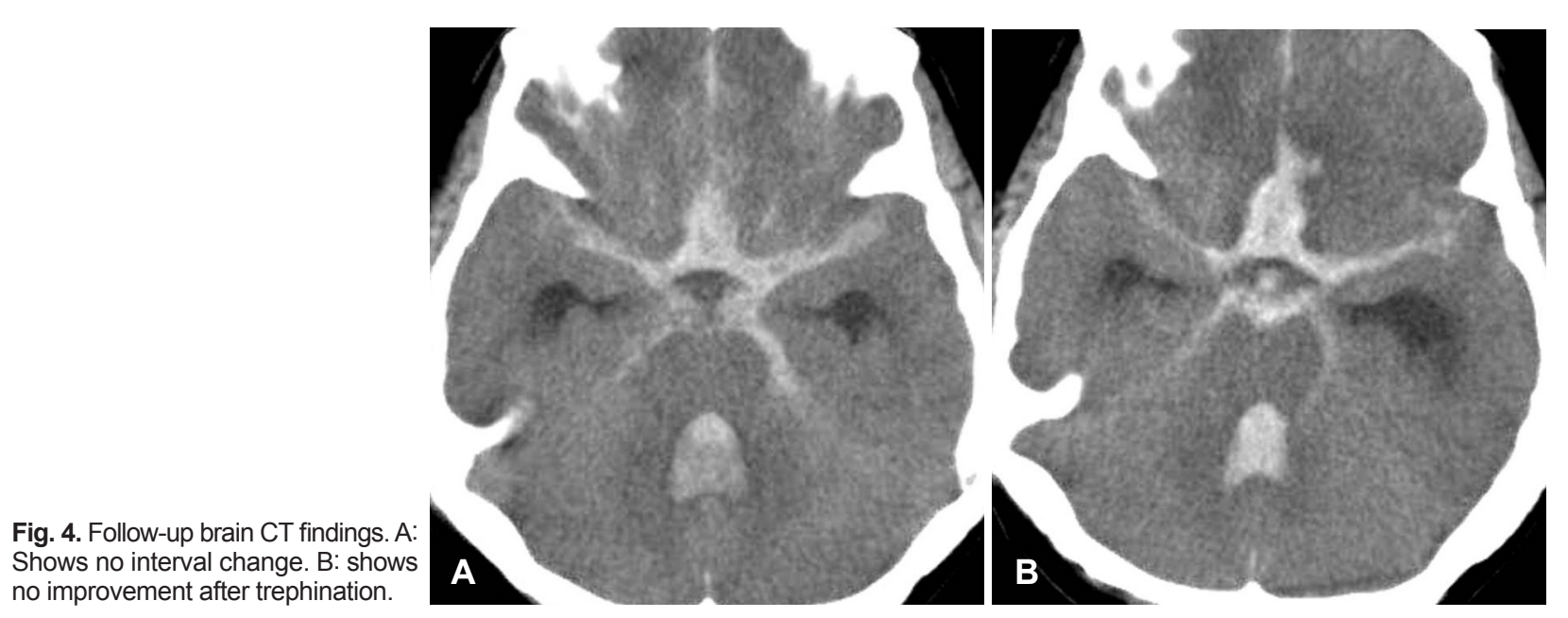

hematoma and a ruptured aneurysm, to the extent that the location of an underlying aneurysm can be relatively well predicted before cerebral angiography. According to previous reports, most hematomas secondary to a ruptured anterior communicating aneurysm are localized in the frontal lobe and ventricular system. ${ }^{2-4)}$ Moreover, although a few basal ganglia hemorrhages are caused by middle cerebral artery aneurysms or internal cerebral artery bifurcation aneurysms, these hemorrhages are rarely caused by a ruptured anterior communicating aneurysm. ${ }^{2,5)}$

Regarding to the pathogenesis in our case, three mechanisms are possible: 1) the aneurysm and the intracerebral hemorrhage (ICH) might have occurred simultaneously but independently of each other; 2) the aneurysmal rupture could have caused a reflex increase in blood pressure and increased intracranial pressure, and caused a hypertensive ICH due to rupture of a penetrating artery weakened by chronic arterial hypertension; 3) a sudden rapid increase in blood pressure could have caused the hypertensive ICH first, and this might then have triggered remote aneurysmal rupture. However, the likelihood of the independent concurrence of aneurysmal rupture and ICH is slight, which adds weight to the other two mechanisms. ${ }^{6)}$

The rupture of an intracranial aneurysm in the subarachnoid compartment immediately causes a life-threatening situation. Sudden death due to a massive increase in intracranial pressure occurs in approximately $10 \%$ of patients (in $40 \%$ of those an aneurysm in the posterior circulation), and another 10 to $20 \%$ of patients as are comatose on admission and require immediate respiratory support. ${ }^{7,8)}$ At the other end of the clinical spectrum, and more commonly, patients present with acute excruciating headache, confusion, or abnormal behavior. Because of the comparatively infrequent presentation of a SAH (approximately 1 in 10000 Americans annually), physicians who encounter patients with a SAH are not attuned to its severity and may find it difficult to recognize its key components. ${ }^{9,10)}$

Patients with an aneurysmal SAH require early repair of the ruptured aneurysm, and in most larger institutions, patients are admitted to a neurologic-neurosurgical intensive care unit and receive aggressive care to prevent further deterioration, which can substantially affect outcome. Furthermore, this care requires a multidisciplinary approach and immediate availability, and thus, it can only be provided by tertiary care medical centers.

We present a fatal case of aneurysmal rupture with SAH 2 weeks after ear surgery. We suggest there is a need to discuss the evaluations required for the diagnosis and management of such patients, and to consider the possibility of aneurysmal rupture after surgery under general anesthesia. Previous reports have addressed the etiology of intracranial hemorrhage after surgery under general anesthesia, and most focused on anesthetic causes, such as, blood pressure and heart rate increases on intubation or anesthetic administration. However, we believe there is a need to focus on guidelines that facilitate early detection and proper management. In the described, warning signs and symptoms were evident immediately after surgery, but brain CT findings were normal and the patient was recovered spontaneously. As a result, we overlooked this case after recovery and consequently lost the patient. Consequently, otologic surgeon should be aware of the possibility of intracerebral hemorrhage after otologic surgical procedures accompanying with general anesthesia. Also, it could be occurred as a delayed fatal complication.

\section{REFERENCES}

1) Hong JH, Jo YH, Choi HJ, Cho TK. A case of spontaneous intracerebral hemorrhage during ear surgery under general anesthesia. J Ko- 
rean Neurosurg Soc 1997;26:136-40

2) Masson RL Jr, Day AL. Aneurysmal intracerebral hemorrhage. Neurosurg Clin N Am 1992;3:539-50.

3) Pasqualin A, Bazzan A, Cavazzani P, Scienza R, Licata C, Da Pian R. Intracranial hematomas following aneurysmal rupture: experience with 309 cases. Surg Neurol 1986;25:6-17.

4) Tokuda Y, Inagawa T, Katoh Y, Kumano K, Ohbayashi N, Yoshioka H. Intracerebral hematoma in patients with ruptured cerebral aneurysms. Surg Neurol 1995;43:272-7.

5) Silver AJ, Pederson ME Jr, Ganti SR, Hilal SK, Michelson WJ. CT of subarachnoid hemorrhage due to ruptured aneurysm. AJNR Am J Neuroradiol 1981;2:13-22.

6) Lee JK, Lee JH, Kim IY, Kim TS, Jung S, Kim JH, et al. Simultaneous occurrence of subarachnoid hemorrhage due to ruptured aneu- rysm and remote hypertensive intracerebral hemorrhage: case report. J Korean Med Sci 2002;17:144-6.

7) Schievink WI, Wijdicks EF, Parisi JE, Piepgras DG, Whisnant JP. Sudden death from aneurysmal subarachnoid hemorrhage. Neurology 1995;45:871-4.

8) Huang J, van Gelder JM. The probability of sudden death from rupture of intracranial aneurysms: a meta-analysis. Neurosurgery 2002; 51:1101-5; discussion 1105-7.

9) Ingall TJ, Whisnant JP, Wiebers DO, O'Fallon WM. Has there been a decline in subarachnoid hemorrhage mortality? Stroke 1989;20:71824.

10) Wijdicks EF, Kallmes DF, Manno EM, Fulgham JR, Piepgras DG. Subarachnoid hemorrhage: neurointensive care and aneurysm repair. Mayo Clin Proc 2005;80:550-9. 